\title{
Epidemiologia do traumatismo raquimedular por projéteis de armas de fogo em um hospital de referência no estado do Pará
}

\section{Epidemiology of Spinal Cord Injury by Firearm in a Reference Hospital in the State of Pará}

\author{
Edmundo Luis Rodrigues Pereira ${ }^{1} \quad$ Alzira Leite Gomes ${ }^{2}$ Daniella Brito Rodrigues ${ }^{2}$ \\ ${ }^{1}$ Neurocirurgião, Mestrado em Neurociências pela Universidade \\ Federal do Pará (UFPA), Belém, PA, Brasil \\ ${ }^{2}$ Acadêmica de Medicina da Universidade do Estado do Pará (UEPA), \\ Belém, PA, Brasil \\ Arq Bras Neurocir 2015;34:13-19. \\ Address for correspondence Edmundo Luis Rodrigues Pereira, MSc, \\ Rua dos Pariquis, 1838/802, Jurunas, Belém, PA, Brasil, CEP 66033-590 \\ (e-mail: eluis@ufpa.br). \\ Universidade do Estado do Pará, Belém, PA, Brasil e Universidade \\ Federal do Pará, Belém, PA, Brasil
}

\section{Resumo \\ Palavras-chave \\ - traumatismos da medula espinhal \\ - ferimentos penetrantes \\ - medula espinhal \\ - epidemiologia}

Abstract
Objetivo O presente trabalho objetivou descrever os aspectos clínicos e epidemiológicos do traumatismo raquimedular causado por ferimentos de armas de fogo em pacientes internados no Hospital Metropolitano de Urgências e Emergências, em Belém/PA, no período de janeiro de 2008 a dezembro de 2013.

Método Estudo transversal, retrospectivo e descritivo, com base na análise de prontuários de 89 pacientes vítimas de trauma raquimedular por ferimento por arma de fogo, de acordo com os dados obtidos no setor de registro hospitalar.

Resultados Os traumas decorrentes de projéteis de armas de fogo foram responsáveis por 9,2\% dos traumas raquimedulares, apresentando uma média de 14,8 novos casos por ano; $86,5 \%$ destes eram pacientes do sexo masculino, $73,1 \%$ apresentavam menos de 30 anos. O segmento cervical foi o mais acometido (46\%) e 51,8\% dos pacientes foram classificados como ASIA A. A maior parte foi tratada conservadoramente $(75,3 \%)$ e $56,2 \%$ dos pacientes evoluíram com alguma complicação durante a internação, sendo mais prevalentes as complicações infecciosas (45\%). A taxa de óbito foi de $18 \%$.

Conclusão O trauma raquimedular decorrente de ferimentos de armas de fogo nos pacientes atendidos no Hospital Metropolitano de Urgências e Emergências acomete principalmente homens jovens. O nível cervical foi o mais acometido e a classificação ASIA A foi a mais prevalente. O tratamento conservador foi adotado na maioria dos casos, sendo que mais da metade dos pacientes evoluiu com complicações, especialmente infecciosas, enquanto uma significativa parcela evoluiu para óbito.

Objective This study aimed to describe the clinical and epidemiological aspects of Spinal Cord Injury caused by firearms in patients admitted to the Metropolitan Urgency and Emergency Hospital, Belém, Pará, from January 2008 to December 2013. received

October 10, 2014

accepted

November 1, 2014
DOI http://dx.doi.org/ 10.1055/s-0035-1547384. ISSN 0103-5355.
Copyright $@ 2015$ by Thieme Publicações License terms

Ltda, Rio de Janeiro, Brazil 


\author{
Keywords \\ - spinal cord injuries \\ - wounds penetrating \\ - spinal cord \\ - epidemiology
}

Methods It was developed a cross-sectional, retrospective and descriptive study, based on analysis of medical records of 89 patients diagnosed with Spinal Cord Injury by firearm's injury.

Results It was observed that firearm's injury accounted for 9,2\% of Spinal Cord Injury, with a average of 14,8 news cases per year; $86,5 \%$ of these were male; $73,1 \%$ had less than thirty years. The cervical segment was the most affected $(46 \%)$ and $51,8 \%$ of the pacients were classified as ASIA A. The most were treated conservatively $(75,3 \%)$ and $56,2 \%$ had some complication during the hospitalization, the infectious complications were the more frequent (45\%). The dead rate was $18 \%$.

Conclusion Thus, this study concluded that the $\mathrm{SCl}$ by firearm's injury in patients treated at Metropolitan Urgency and Emergency Hospital affects mainly young men, the traumas occurred mainly in the cervical segment and the classification ASIA A was the most prevalent. Conservative treatment was adopted in most cases and more than half of the patients developed complications, especially infectious, while a significant portion evolve to death.

\section{Introdução}

O aumento progressivo da incidência e gravidade do trauma raquimedular (TRM) por projétil de arma de fogo (PAF) nos últimos anos tem sido motivo de grande preocupação, por espelhar desequilíbrio social que afeta a todos, principalmente jovens em idade laboral, devendo ser considerado pelos órgãos públicos como grave problema de saúde pública. ${ }^{1-3}$ Ademais, o tipo de lesão neural decorrente deste mecanismo costuma acarretar com mais frequência uma anormalidade medular completa, com piores prognósticos em relação às demais causas de lesão espinhal. ${ }^{4,5}$

As lesões por arma de fogo representam, entre as internações por causas externas, a maior taxa de mortalidade, com aproximadamente 10 óbitos por 100 internações e com custo $34 \%$ mais elevado em relação aos outros tipos de agressões. ${ }^{6}$ Nos casos de TRM por ferimentos por arma de fogo (FAF), as taxas de mortalidade variam de 3 a $18,5 \%$, sendo maiores nos pacientes com lesão cervical e ASIA A. A idade avançada, comorbidades preexistentes, e lesão neurológica completa foram consideradas significativos fatores de risco para mortalidade intra-hospitalar. ${ }^{7-10}$

O conhecimento sobre dados epidemiológicos é essencial para se propor medidas de prevenção e concentrar recursos técnicos e humanos em serviços de referência ao atendimento e tratamento dos pacientes com lesão medular. ${ }^{11,12}$ As informações mais detalhadas acerca da etiologia são importantes para gerar intervenções de acordo com as necessidades da região. ${ }^{12,13}$ Além disso, a importância de trabalhos epidemiológicos sobre pacientes com lesão medular aguda torna-se ainda mais evidente quando observamos que a única forma de melhorar a qualidade de vida da população e diminuir os custos com estes pacientes é evitar que as complicações aconteçam. ${ }^{14}$

Diante do exposto, o presente estudo se propõe a analisar as características clínicas e epidemiológicas das vítimas de TRM decorrente de ferimentos de armas de fogo atendidas no
Hospital Metropolitano de Urgências e Emergências (HMUE), hospital de referência em traumatologia e no atendimento a este tipo de lesão no estado do Pará, no período de janeiro de 2008 a dezembro de 2013.

\section{Método}

Este trabalho foi realizado de acordo com as normas de pesquisa envolvendo seres humanos do Conselho Nacional de Saúde (Resolução 196/96) e com os preceitos da Declaração de Helsinque e do Código de Nuremberg, sendo aprovado pelo Comitê de Ética da Universidade do Estado do Pará e pela direção do Hospital Metropolitano de Urgências e Emergências do estado do Pará.

A presente pesquisa é caracterizada como um estudo transversal, retrospectivo, observacional e descritivo. Foi desenvolvida a partir da análise de prontuários, nos quais foram observadas as variáveis: idade, sexo, procedência, profissão, déficits neurológicos segundo a escala da ASIA, tratamento instituído, lesões associadas, complicações sistêmicas e óbito. Este protocolo foi elaborado com base nos estudos de Barros Filho et al., ${ }^{15}$ Blanes et al., ${ }^{16}$ Pereira e Jesus, ${ }^{12}$ Inaba et al. ${ }^{17}$ e Chen et al. ${ }^{13}$

Foram classificados como lesões associadas, os ferimentos por arma de fogo localizados em regiões anatômicas do corpo além da medula espinhal e lesões decorrentes do mecanismo do trauma como fraturas ortopédicas, lesões de nervos periféricos ou de vasos, etc. Desta maneira, as lesões associadas foram divididas nos seguintes subgrupos: traumatismo cranioencefálico, trauma bucomaxilofacial, trauma torácico, trauma abdominal, trauma pélvico, lesão de plexo braquial, lesões ortopédicas e outras. Como lesões ortopédicas foram consideradas: fraturas de mão, fraturas de punho, fraturas de clavícula, fraturas de costelas, fraturas de arcos costais, fraturas de membros superiores e membros inferiores. No subgrupo "outras" estão inclusas: lesões de globo ocular, diafragma, pescoço, dorso e laringe. 
Tabela 1 Distribuição dos traumas raquimedulares (TRM) por causas gerais e dos traumas raquimedulares por ferimento de arma de fogo (TRM/FAF), de acordo com o ano, em pacientes atendidos no HMUE, Belém/PA, no período de 2008 a 2013

\begin{tabular}{|l|l|l|l|}
\hline ANO & TRM & TRM/FAF & $\%^{*}$ \\
\hline 2008 & 167 & 23 & 13,8 \\
\hline 2009 & 174 & 13 & 7,5 \\
\hline 2010 & 131 & 13 & 9,9 \\
\hline 2011 & 156 & 15 & 9,6 \\
\hline 2012 & 149 & 6 & 4,0 \\
\hline 2013 & 195 & 19 & 9,7 \\
\hline TOTAL & 972 & 89 & $9,2 \%$ \\
\hline
\end{tabular}

Fonte: Banco de dados da pesquisa.

$\left(^{*}\right)$ Porcentagem de TRM por FAF em relação ao total de TRM em cada período.

Em relação à procedência, os pacientes foram organizados, de acordo com a cidade de origem, entre as regiões administrativas do estado do Pará, de acordo com a divisão administrativa utilizada pelo Ministério Público do Estado.

$\mathrm{O}$ trajeto do projétil foi classificado em intracanal e extracanal. No subgrupo extracanal, estão inclusos os projéteis transfixantes e os alojados no corpo e disco vertebrais.

Foi estudada uma casuística de 89 pacientes, referente ao total de pacientes com traumatismos raquimedulares decorrentes de ferimentos por armas de fogo atendidos no período de janeiro de 2008 a dezembro de 2013.

Foram incluídos no estudo pacientes de ambos os sexos, sem distinções quanto à etnia, grau de escolaridade, níveis de renda ou estado civil. Estes pacientes haviam sido diagnosticados com traumatismo raquimedular e ferimentos por armas de fogo (CID 10: S22.0, S23.1, S24, S24.0, S12.7, S14.2, S32, S33, X93). Foram excluídos pacientes cujo prontuário foi preenchido com dados insuficientes para análise e aqueles pacientes com traumatismo raquimedular decorrente de outras causas.

Os dados obtidos foram organizados em um banco de dados, sendo posteriormente submetidos à análise estatística descritiva. Os softwares Microsoft Office Excel 2007 ${ }^{\mathrm{TM}}$ e Bioestat $5.0^{\mathrm{TM}}$ foram utilizados para análise dos dados e confecção de tabelas.

\section{Resultados}

Foram analisados 89 pacientes atendidos no serviço de neurocirurgia do Hospital Metropolitano de Urgências e Emergências no período de janeiro de 2008 a dezembro de 2013. Ocorreram 972 casos de traumatismo raquimedular no período da pesquisa, sendo 89 destes decorrentes de projéteis de armas de fogo (PAF), correspondendo, portanto, a 9,2\% do total de casos (-Tabela 1 e - Fig. 1).

A região metropolitana de Belém foi a mais incidente com $50,6 \%$ dos casos, seguida pelo nordeste do estado (34,8\%), Tocantins e Marajó (9\%), sudeste (4,5\%) e não informada em 1,1\% dos casos (-Fig. 2).

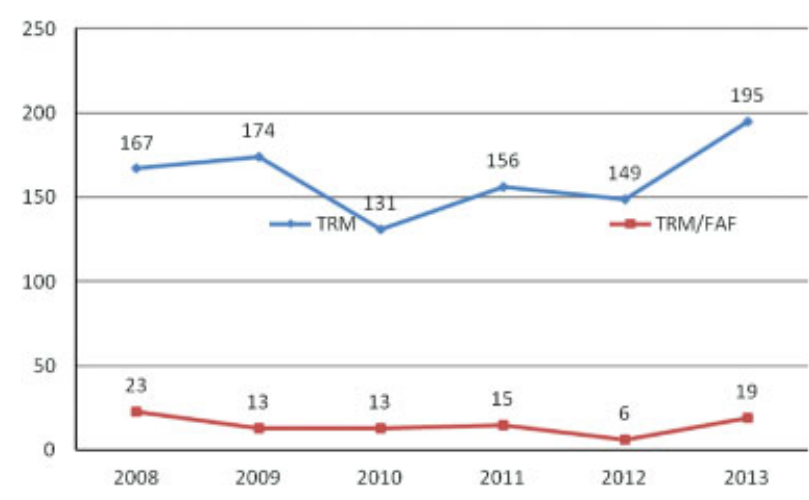

Fig. 1 Distribuição dos traumas raquimedulares (TRM) por causas gerais e dos traumas raquimedulares por ferimento de arma de fogo (TRM/FAF), de acordo com o ano, em pacientes atendidos no HMUE, Belém/PA, no período de 2008 a 2013. Fonte: Banco de dados da pesquisa.

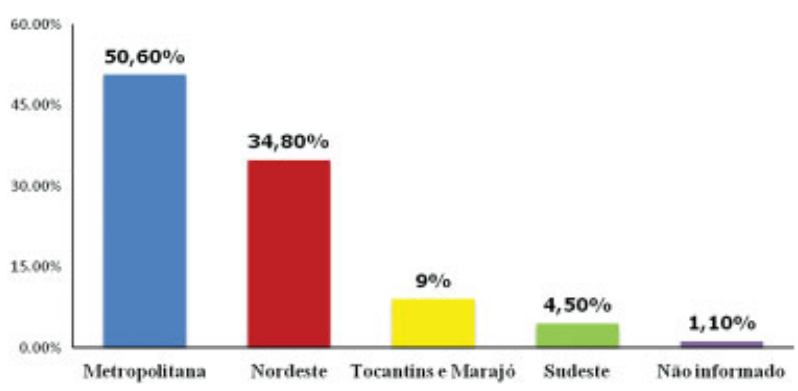

Fig. 2 Distribuição dos pacientes vítimas de trauma raquimedular por ferimento de arma de fogo atendidos no HMUE de acordo com o trajeto do projétil, Belém/PA, no período de 2008 a 2013. Fonte: Banco de dados da pesquisa.

O sexo masculino foi o mais prevalente (86,5\%). A idade média foi de 26,5 anos, sendo 3 anos a idade mínima e 55 anos a idade máxima. Maior prevalência foi encontrada na faixa etária dos 21 a 30 anos (48,4\%) (-Fig. 3).

O segmento cervical foi o mais acometido (46\%), seguido pelo torácico (27\%) e lombar (5,6\%); entre as lesões múltiplas encontradas, $18 \%$ afetavam os segmentos cervical e torácico e 3,4\% os segmentos torácico e lombar (-Fig. 4).

A maioria dos pacientes foi classificada como ASIA A (52,3\%), enquanto $25 \%$ corresponderam ao ASIA E, seguidos pelo ASIA D (10,2\%), ASIA C (9,1\%) e ASIA B (3,4\%) (-Fig. 5).

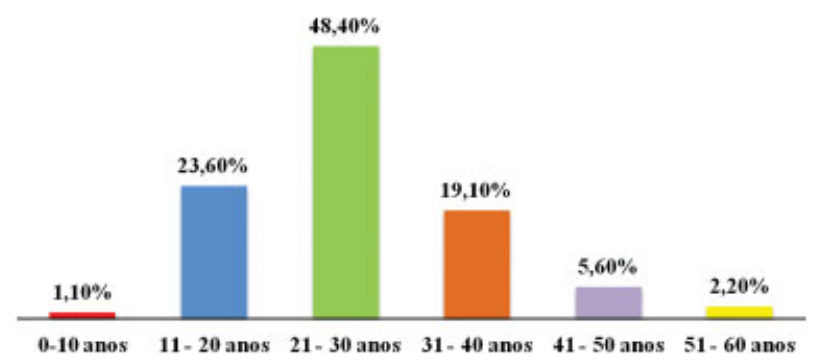

Fig. 3 Distribuição de acordo com a idade dos pacientes vítimas de trauma raquimedular por ferimento de arma de fogo atendidos no HMUE, Belém/PA, no período de 2008 a 2013. Fonte: Banco de dados da pesquisa. 


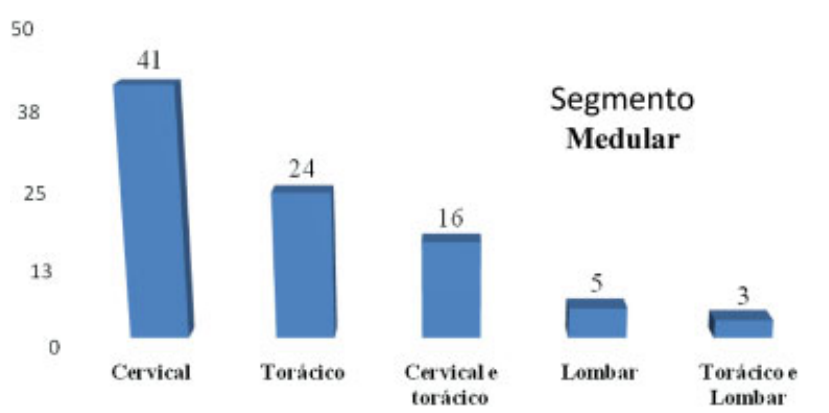

Fig. 4 Distribuição de acordo com o segmento medular afetado dos pacientes vítimas de trauma raquimedular por ferimento de arma de fogo atendidos no HMUE, Belém/PA, no período de 2008 a 2013. Fonte: Banco de dados da pesquisa.

Dos pacientes estudados, 68 (76,4\%) apresentaram outras lesões associadas ao TRM, sendo o traumatismo cranioencefálico e o trauma torácico as principais associações (-Fig. 6).

O tratamento conservador foi adotado em $75,3 \%$ dos pacientes. Quanto ao trajeto do projétil, em $60,7 \%$ dos pacientes o projétil percorreu um trajeto intracanal. Entre estes pacientes, $37 \%$ necessitaram de abordagem cirúrgica.

Cerca de metade dos pacientes (56,2\%) apresentou algum tipo de complicação durante a internação. 0 tempo de internação foi em média 21,3 dias, sendo que 59,6\% ficaram internados por até 14 dias. A taxa de mortalidade foi de $18 \%$ e $87,5 \%$ que evoluíram para óbito eram classificados como ASIA A (-Fig. 7).

\section{Discussão}

Poucos eventos no campo da neurocirurgia requerem do especialista tanto empenho e atenção quanto o trauma raquimedular, em especial aqueles decorrentes de ferimentos por arma de fogo. Por ser de instalação súbita e inesperada, torna-se um infortúnio de proporções desastrosas ao doente e a sua família, além de promover altos custos hospitalares à sociedade. ${ }^{14}$

Em nosso estudo, do total de 972 casos de TRM atendidos no período de 2008 a 2013 no Hospital Metropolitano de Urgência e Emergência, os ferimentos por arma de fogo (FAF) foram responsáveis por 89 casos $(9,2 \%)$, apresentando uma

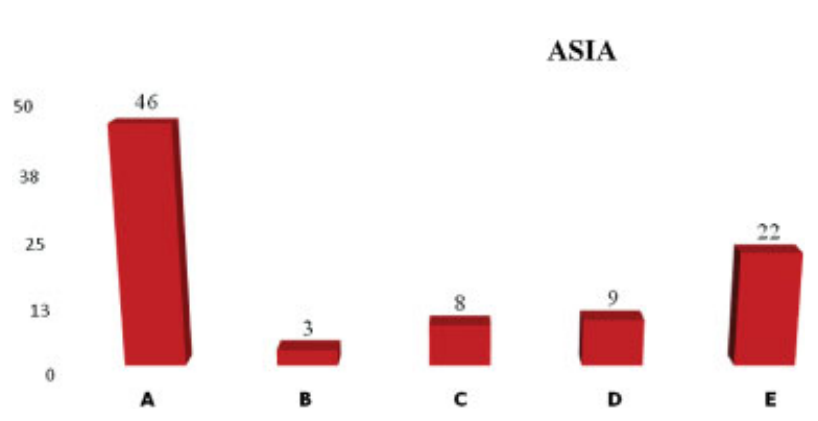

Fig. 5 Distribuição de acordo com a escala da ASIA dos pacientes vítimas de trauma raquimedular por ferimento de arma de fogo atendidos no HMUE, Belém/PA, no período de 2008 a 2013. Fonte: Banco de dados da pesquisa.

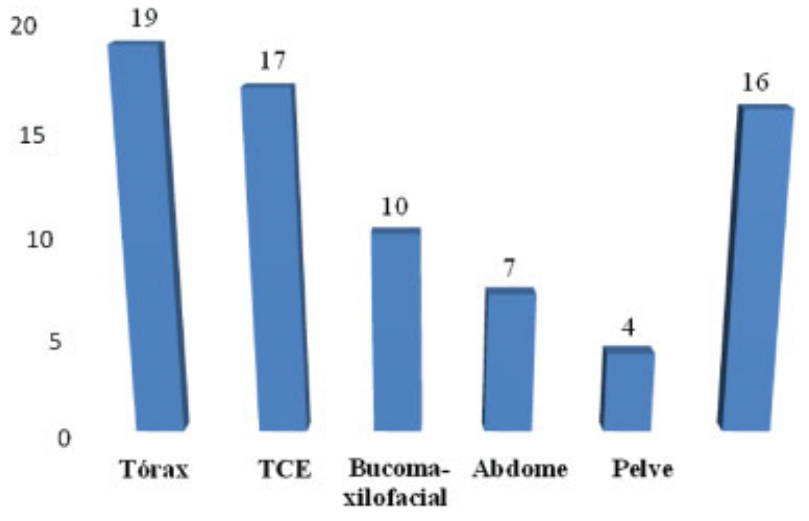

Fig. 6 Distribuição dos pacientes vítimas de trauma raquimedular por ferimento de arma de fogo atendidos no HMUE de acordo com as lesões associadas, Belém/PA, no período de 2008 a 2013. Fonte: Banco de dados da pesquisa. Abreviação: TCE, traumas cranioencefálicos.

média 14,8 novos casos por ano (-Tabela 1 e $\boldsymbol{- F i g . ~} \mathbf{1}$ ). Estes resultados estão abaixo da média mundial relatada, na qual os FAF são responsáveis por 13 a $44 \%$ dos casos de TRM. ${ }^{18}$ Porém se aproximam dos resultados encontrados por Campos et al. ${ }^{14}$ em um hospital de referência na região sul de São Paulo, que evidenciaram os FAF como responsáveis por $7 \%$ dos casos de TRM, e estão acima do percentual encontrado por Pereira et al. ${ }^{19}$ em Recife, que foi de $4,76 \%$.

Quando se avalia a procedência dos pacientes estudados, $50,6 \%$ ( Fig. 2) eram procedentes da região metropolitana de Belém, que conta com cerca de 2,1 milhões de habitantes. Trabalhos anteriores sobre TRM no estado do Pará20,21 encontraram 53,75\% dos pacientes atendidos em 2002 e 61,6\% atendidos em 2003 procedentes do interior do estado.

Em relação à idade, a maioria dos pacientes possuía de 21 a 30 (-Fig. 3), o que está de acordo com a maioria dos resultados obtidos no restante da literatura mundial, ${ }^{9,13,14,22-25}$ particularmente em estudos epidemiológicos realizados em países em desenvolvimento. Reiterando que os adultos jovens, principalmente na faixa etária de 21 a 31 anos de idade, são o grupo etário de maior risco para lesões medulares, especialmente as decorrentes de ferimentos por arma de fogo. Conforme observado por Pereira e

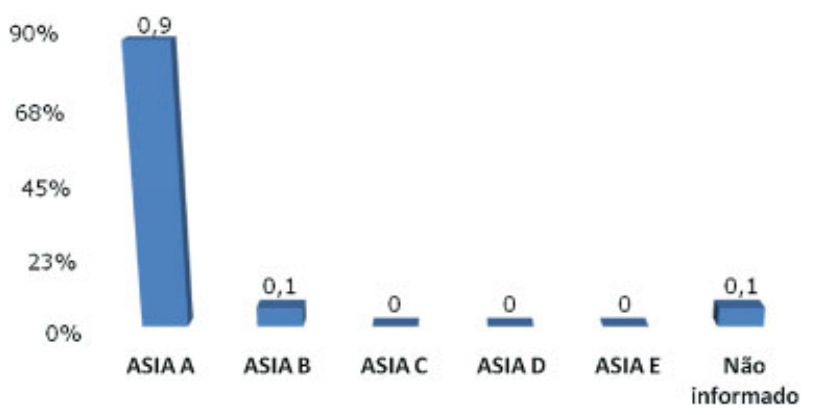

Fig. 7 Frequência de óbitos, de acordo com a classificação na escala da ASIA, entre os pacientes vítimas de trauma raquimedular por ferimento de arma de fogo atendidos no HMUE, Belém/PA, no período de 2008 a 2013. Fonte: Banco de dados da pesquisa. 
Jesus, ${ }^{12}$ esta faixa etária tende a apresentar maiores índices por se expor mais a comportamentos de risco, como a prática de atividades ilícitas, discussões em festas e sob ingestão de bebidas alcoólicas, e hábitos mais noturnos, período em que as taxas de violência tendem a ser mais elevadas. Vários trabalhos elucidam ainda o fato de que estes jovens correspondem justamente à parcela economicamente ativa da população, e que grande parte evolui com déficits neurológicos irreversíveis, representando, dessa forma, não só uma grande perda de mão de obra, mas um prejuízo ainda maior à sociedade, gerando altos custos com tratamento e reabilitação. $^{9}$

No que se refere ao sexo, a grande maioria dos pacientes pertenciam ao sexo masculino, o que também está de acordo com o restante dos trabalhos, com a frequência de vítimas do sexo masculino por ferimentos por armas de fogo na coluna variando entre 78 e $94 \%$ na literatura. ${ }^{9,14,18,24-26}$ Estes dados podem ser justificados pelo fato de os homens se exporem mais aos comportamentos de risco para ferimentos por arma de fogo já citados anteriormente.

Analisando os segmentos medulares afetados (-Fig. 4), observou-se que o segmento cervical foi o mais afetado, respondendo por cerca de metade dos casos, seguido pelo seguimento torácico. A literatura demonstra que os traumas raquimedulares em geral tendem a afetar mais a porção cervical da coluna, ${ }^{25,27}$ porém, quando se trata de TRM por FAF, os trabalhos apontam o segmento torácico como o mais acometido. ${ }^{18,27,28}$ No entanto, os trabalhos são unânimes em apontar que os segmentos cervicais e torácicos são os mais afetados tanto nas lesões medulares por causas gerais quanto nas por FAF.

Os dados referentes ao segmento medular mais afetado são importantes para predizer que tipo de complicações são mais prováveis para cada paciente e assim podem fornecer subsídios em relação aos materiais hospitalares de maior demanda para estes pacientes. As lesões na coluna cervical, por exemplo, são frequentemente associadas a lesões das vias aéreas e podem requerer entubação de emergência. ${ }^{18}$ Além disso, estas injúrias cervicais podem estar relacionadas com morte súbita, síndrome de Brown-Sequard e paralisia cruzada. ${ }^{27}$ Outro dado interessante é que estas lesões são estáveis em sua maioria, apresentando menor necessidade de abordagem cirúrgica. A razão para a estabilidade de lesões cervicais de ferimento por arma de fogo em comparação com traumas fechados é que o complexo ligamentoso permanece intacto na maioria dos casos, apesar da destruição da coluna anterior. $^{18}$

Quando se avalia a classificação da American Spinal Injury Association - ASIA ( - Fig. 5), observa-se que pouco mais da metade dos pacientes foram classificados como ASIA A, o que está de acordo com dados observados nos trabalhos de Barros Filho et al. ${ }^{18}$ em uma revisão de literatura e de Koch et al. ${ }^{29}$ em um hospital do Paraná. Neste último, os autores observaram ainda que o FAF, entre todas as etiologias de lesões medulares verificadas, foi o mecanismo com maior porcentagem de lesões neurológicas. É importante destacar que uma das variáveis mais aceitas pela literatura como fator prognóstico é a apresentação clínica dos pacientes à admis- são, sendo que as lesões completas (ASIA A) apresentam muito baixo potencial de recuperação, enquanto as incompletas e de cauda equina apresentam potencial de recuperação de $47 \%$ e $86 \%$, respectivamente. ${ }^{9}$

Em relação às lesões associadas, os traumas de tórax e os traumas cranioencefálicos (TCE) foram os mais frequentes (-Fig. 6). Morais et al. ${ }^{30}$ também concluíram que estes eram os traumas mais frequentes. Le Roux et al. ${ }^{31}$ também consideraram o trauma torácico como preponderante, seguido pelas lesões ortopédicas. Este predomínio no presente estudo pode ser explicado pela maior incidência de lesões cervicais e torácicas, o que, anatomicamente, favorece lesões associadas torácicas e cranianas.

Na maioria dos pacientes, o projétil assumiu um trajeto intracanal. No estudo conduzido por Le Roux e Dunn, ${ }^{31}$ o projétil foi intracanal em $25 \%$ dos casos. Incidências ainda menores foram avaliadas por Araújo Júnior et al. ${ }^{9}$ e Barros Filho. ${ }^{15}$ É válido destacar que a descompressão e a retirada de projéteis intracanais em T12 e em níveis abaixo podem melhorar a função motora, justificando a indicação cirúrgica. $^{32}$

A maior parte dos pacientes foi tratada conservadoramente, o que corrobora os estudos de Araújo Júnior et al. ${ }^{9}$ e Benato et al., ${ }^{33}$ mas diverge dos trabalhos de Koch et al. ${ }^{29} \mathrm{e}$ Beaty et al., ${ }^{5}$ que encontraram taxas maiores de intervenção cirúrgica. Existe consenso na literatura de que as lesões por FAF são de tratamento conservador em grande parte dos casos, seja porque as fraturas causadas são geralmente estáveis ou porque a descompressão medular nem sempre é indicada. ${ }^{18,34-36}$

Entre os pacientes tratados cirurgicamente, apenas um não apresentava o projétil com trajeto intracanal. Dados semelhantes foram encontrados por Benato et al., ${ }^{33}$ que tratou cirurgicamente todos os projéteis que estavam dentro do canal medular. Embora as indicações de cirurgia sejam controversas, a literatura descreve como principais indicações para laminectomia descompressiva com retirada do projétil: déficit neurológico progressivo em um paciente com lesão medular incompleta, migração do projétil intracanal, fístula dural, instabilidade do segmento atingido, suspeita de intoxicação por chumbo, e pacientes com déficit neurológico parcial, especialmente aqueles com síndrome da cauda equina, com o comprometimento do canal vertebral pelos fragmentos ósseos ou metálicos. ${ }^{18,37,38}$

No entanto, a única indicação absoluta de cirurgia na coluna vertebral devido a lesões por PAF é a presença de fístula liquórica ou a presença de progressão documentada do déficit neurológico associada com compressão de elementos neurais. $^{18,37}$

Neste estudo, mais da metade dos pacientes apresentaram complicações, o que concorda com a maior parte da literatura vigente. Estas elevadas taxas de complicação podem ser reflexo das dificuldades locais de acesso à assistência pública de saúde no estado, decorrentes da carência de mais centros especializados em trauma, bem como da falta de agilidade no atendimento a estas vítimas e do prolongado tempo de internação. 
O tempo médio de internação foi de 21,3 dias, resultado maior do que o encontrado por autores como Siscão et al. ${ }^{39}$ e Araújo Júnior et al., ${ }^{9}$ fato este que pode ser justificado pelo elevado índice de complicações secundárias à lesão medular.

O óbito ocorreu em $18 \%$ dos pacientes estudados. Mortalidade significativamente mais elevada do que a encontrada por outros estudos, ${ }^{10,40,41}$ porém em concordância com os trabalhos de Araújo Júnior et al. ${ }^{9}$ em Curitiba e Azevedo Filho et al. ${ }^{42}$ em Recife, que encontraram $18,5 \%$ e $14,2 \%$ de mortalidade, respectivamente.

A maioria dos óbitos ocorreu em pacientes ASIA A (-Fig. 7), o que corrobora os estudos de Grossman et al., ${ }^{10}$ em que todos os pacientes que evoluíram para óbito haviam sido classificados como ASIA A. Azevedo Filho et al. ${ }^{42} \mathrm{e}$ Barros Filho et al. ${ }^{18}$ também concluíram que a mortalidade em pacientes vítimas de TRM por FAF aumenta de acordo com a severidade do déficit neurológico.

Destarte, é notório que a violência urbana criou novos perfis de mortalidade e comorbidades entre os indivíduos jovens em faixa etária produtiva, sendo crucial reconhecer as características distintas dessa entidade para otimizar a utilização de recursos de assistência médica e intervir de modo a alterar o prognóstico desses pacientes. Destaca-se, entretanto, que a prevenção ainda é o melhor tratamento dessas causas de morte, devendo ser multifacetada, e que é preciso considerar as características individuais e o contexto social e político da lesão.

\section{Conclusão}

O traumatismo raquimedular por ferimentos de armas de fogo representou 9,2\% das etiologias de trauma raquimedular, sendo mais frequente no sexo masculino, na faixa etária de 21 a 30 anos. A maioria dos casos ocorreu na região metropolitana de Belém. O segmento cervical foi o mais acometido, e a maioria dos pacientes foi classificada como ASIA A, isto é, com lesão completa no momento da admissão hospitalar. Projéteis com acometimento do canal intrarraquiano representam $60,7 \%$ dos casos. Trauma de tórax e trauma cranioencefálico foram as principais lesões associadas. $\mathrm{O}$ tratamento conservador foi o mais empregado, e $56,2 \%$ dos pacientes tiveram complicações clínicas, sendo as infecciosas e pulmonares as mais frequentes. O óbito ocorreu em $18 \%$ dos pacientes, de modo preponderante nas vítimas classificadas como ASIA A.

Conflitos de Interesse

Os autores declaram não haver conflitos de interesse.

\section{Agradecimentos}

Agradecemos ao Dr. José Claúdio Monteiro Rodrigues e ao Dr. Maurício Pinho Lima pelas valiosas contribuições intelectuais a este trabalho.

\section{Referências}

1 Gonçalves AMT, Rosa LN, D’Ângelo CT, et al. Aspectos epidemiológicos da lesão medular traumática na área de referência do Hospital Estadual Mario Covas. Arq Med ABC 2007;32(2):64-66

2 Pimentel MG, Gomes EGF, Gusmão MS, et al. Estudo epidemiológico dos traumas raquimedulares por projétil de arma de fogo no Hospital Geral do Estado da Bahia. Coluna/Columna 2012;11(4): 298-301

3 Botelho RV, Albuquerque LDG, Bastianello R Jr, Arantes AA Jr. Epidemiology of traumatic spinal injuries in Brazil: sistematic review. Arq Bras Neurocir 2014;33(2):100-106

$4 \mathrm{Kim}$ J, Kim JH, Bang MS. A case report of spinal cord injury patient from a high velocity gunshot wound to the lumbar spine. Ann Rehabil Med 2013;37(1):118-122

5 Beaty N, Slavin J, Diaz C, Zeleznick K, Ibrahimi D, Sansur CA. Cervical spine injury from gunshot wounds. J Neurosurg Spine 2014;21(3):442-449

6 Souza ER. Masculinidade e violência no Brasil: contribuições para a reflexão no campo da saúde. Ciênc Saúde Coletiva 2005;10(1): 59-70

7 Furlan JC, Fehlings MG. Cardiovascular complications after acute spinal cord injury: pathophysiology, diagnosis, and management. Neurosurg Focus 2008;25(5):E13

8 Couris CM, Guilcher SJ, Munce SE, et al. Characteristics of adults with incident traumatic spinal cord injury in Ontario, Canada. Spinal Cord 2010;48(1):39-44

9 Araújo FA Júnior, Heinrich CB, et al. Traumatismo raquimedular por ferimento de projétil de arma de fogo: avaliação epidemiológica. Coluna/Columna 2011;10(4):290-292

10 Grossman RG, Frankowski RF, Burau KD, et al. Incidence and severity of acute complications after spinal cord injury. J Neurosurg Spine 2012;17(Suppl 1):119-128

11 Gawryszewski VP, Rodrigues EM. The burden of injury in Brazil, 2003. Sao Paulo Med J 2006;124(4):208-213

12 Pereira CU, Jesus RM. Epidemiology of spinal injury in Aracaju. A prospective series. J Bras Neurocirurg 2011;22(2):26-31

13 Chen Y, Tang Y, Vogel LC, Devivo MJ. Causes of spinal cord injury. Top Spinal Cord Inj Rehabil 2013;19(1):1-8

14 Campos MF, Ribeiro AT, Listik S, Pereira CAB, Sobrinho JA, Rapaport A. Epidemiologia do traumatismo da coluna vertebral. Rev Col Bras Cir 2008;35(2):88-93

15 Barros Filho TE, Oliveira RP, Barros EK, et al. Ferimentos por arma de fogo na coluna vertebral: estudo epidemiológico. Coluna/ Columna 2002;1(2):83-87

16 Blanes L, Lourenço L, Carmagnani MI, Ferreira LM. Clinical and socio-demographic characteristics of persons with traumatic paraplegia living in São Paulo, Brazil. Arq Neuropsiquiatr 2009; 67(2B):388-390

17 Inaba K, Barmparas G, Ibrahim D, et al. Clinical examination is highly sensitive for detecting clinically significant spinal injuries after gunshot wounds. J Trauma 2011;71(3):523-527

18 de Barros Filho TE, Cristante AF, Marcon RM, Ono A, Bilhar R. Gunshot injuries in the spine. Spinal Cord 2014;52(7):504-510

19 Pereira AF, Portela LE, Lima GD, et al. Avaliação epidemiológica das fraturas da coluna torácica e lombar dos pacientes atendidos no Serviço de Ortopedia e Traumatologia do Hospital Getúlio Vargas em Recife/PE. Coluna/Columna 2009;8(4):395-400

20 Souza MF Júnior, Bastos BP, Jallageas DN, Medeiros AA. Perfil epidemiológico de 80 pacientes com traumatismo raquimedular, internados no Hospital do Pronto-Socorro Municipal de Belém, PA, no período de janeiro a setembro de 2002. J Bras Neurocirurg 2002;13(3):92-98

21 Souza MF Júnior, Neves AC, Medeiros AA, Jallageas DN. Características epidemiológicas do trauma raquimedular na Amazônia: Análise prospectiva de 250 casos. J Bras Neurocir 2003;14((3)): 97-104 
22 Flores LP, Nascimento Filho JS, Pereira Neto A, Suzuki K. Prognostic factors related to gunshot wounds to the spine in patients submitted to laminectomy. Arq Neuropsiquiatr 1999;57(3B): 836-842

23 Pirouzmand F. Epidemiological trends of spine and spinal cord injuries in the largest Canadian adult trauma center from 1986 to 2006. J Neurosurg Spine 2010;12(2):131-140

24 Brito LM, Chein MB, Marinho SC, Duarte TB. Epidemiological evaluation of victims of spinal cord injury. Rev Col Bras Cir 2011;38(5):304-309

25 Yang R, Guo L, Wang P,et al. Epidemiology of spinal cord injuries and risk factors for complete injuries in Guangdong, China: a retrospective study. PLoS One 2014;9(1):e84733

26 Nogueira PC, Cakiri MHL, Haas VJ. Perfil de pacientes com lesão traumática da medula espinhal e ocorrência de úlcera de pressão em um hospital universitário. Rev Latino-Am Enfermagem 2006; 14(3):33-38

27 Seçer M, Ulutaş M, Yayla E, Cınar K. Upper cervical spinal cord gunshot injury without bone destruction. Int J Surg Case Rep 2014;5(3):149-151

28 Jaiswal M, Mittal RS. Concept of gunshot wound spine. Asian Spine J 2013;7(4):359-364

29 Koch A, Graells XS, Zaninelli EM. Epidemiologia de fraturas da coluna de acordo com o mecanismo de trauma: análise de 502 casos. Coluna/Columna 2007;6(1):18-23

30 Morais DF, Melo Neto JS, Spotti AR, Tognola WA. Preditores de complicações clínicas em pacientes com trauma raquimedular. Coluna/Columna 2014;13(2):139-142

31 Le Roux JC, Dunn RN. Gunshot injuries of the spine-a review of 49 cases managed at the Groote Schuur Acute Spinal Cord Injury Unit. S Afr J Surg. 2005;43(4):165-168

32 Moon E, Kondrashov D, Hannibal M, Hsu K, Zucherman J. Gunshot wounds to thespine: literature review and report on a migratory intrathecal bullet. Am JOrthop (Belle Mead NJ) 2008;37(3): E47-51

33 Benato ML, Zaninelli EM, Graells XS. Avaliação da incidência das lesões por arma de fogo da coluna vertebral. Coluna/Columna 2007;6(3):155-161

34 Bono CM, Heary RF. Gunshot wounds to the spine. Spine J 2004; $4(2): 230-240$

35 Waters RL, Sie IH. Spinal cord injuries from gunshot wounds to the spine. Clin Orthop Relat Res 2003;(408):120-125

36 Bordon G, Burguet Girona S. Gunshot wound in lumbar spine with intradural location of a bullet. Case Rep Orthop 2014; 2014:698585

37 Kumar A, Pandey PN, Ghani A, Jaiswal G. Penetrating spinal injuries and their management. J Craniovertebr Junction Spine 2012;(2):57-61

38 Sidhu GS, Ghag A, Prokuski V, Vaccaro AR, Radcliff KE. Civilian gunshot injuries of the spinal cord: a systematic review of the current literature. Clin Orthop Relat Res 2013;471(12): 3945-3955

39 Siscão MP, Pereira C, Arnal RLC, Foss MHD A, Marino LHC. Trauma Raquimedular: caracterização em um Hospital Público. Arq Ciênc Saúde 2007;14(3):145-147

40 Boakye M, Patil CG, Santarelli J, Ho C, Tian W, Lad SP. Laminectomy and fusion after spinal cord injury: national inpatient complications and outcomes. J Neurotrauma 2008;25(3):173-183

41 Kattail D, Furlan JC, Fehlings MG. Epidemiology and clinical outcomes of acute spine trauma and spinal cord injury: experience from a specialized spine trauma center in Canada in comparison with a large national registry. J Trauma 2009;67(5): 936-943

42 Azevedo-Filho HRC, Martins C, Carneiro-Filho GS, Azevedo R, Azevedo F. Gunshot wounds to the spine: study of 246 patients. Neurosurg Quart 2001;11:199-208 